Eni Devay de Freitas 1

\title{
Evolução e distribuição espacial da mortalidade por causas externas em Salvador, Bahia, Brasil
}

\author{
Trends and spatial distribution \\ of mortality from external causes \\ in Salvador, Bahia State, Brazil
}

Jairnilson Silva Paim 1

Lígia Maria Vieira da Silva 1

Maria da Conceição Nascimento Costa 1

\footnotetext{
1 Instituto de Saúde Coletiva Universidade Federal da Bahia. Rua Padre Feijó 29 4o andar, Salvador, BA 40110-070, Brasil. jairnil@ufba.br
}

\begin{abstract}
Since 1980, external causes (ICD-9 E800-E999) have been ranked as the second leading causal group for mortality in Brazil, thus becoming a major public health problem. This study aimed to describe spatial distribution trends for violent deaths in the urban setting of Salvador, a city in Northeast Brazil, for the years 1988, 1991, and 1994. An ecological study was conducted, and mortality data were obtained from death certificates and the archives of the Institute for Forensic Medicine. There was an increase of 34.6\% in the number of deaths from external causes between 1988 and 1994. The highest mortality rates were among men from 20 to 29 years of age (from 192.0 to 262.0/100,000) and those 65 years and over (from 188.7 to 258.1/100,000). Homicides were the leading cause of violent deaths in about $75.0 \%$ of neighborhoods. The authors discuss the need for comprehensive public policies and an interdisciplinary approach to elucidate the causes and deal with the problem of violence.
\end{abstract}

Key words Sudden Death; Mortality; Violence; Spatial Analysis

Resumo A partir de 1980, as mortes violentas vêm se destacando como segunda principal causa de óbito no Brasil, tornando-se um importante problema de saúde pública. Este trabalho tem como objetivo descrever a evolução e a distribuição espacial das mortes violentas no espaço urbano de Salvador, uma cidade do Nordeste do Brasil, nos anos de 1988, 1991 e 1994. Trata-se de um estudo ecológico, cujos dados de mortalidade foram obtidos das Declarações de Óbito e dos arquivos do Instituto Médico Legal. Observou-se um aumento de 34,6\% no número de mortes violentas entre os anos de 1988 e 1994. As maiores taxas de mortalidade ocorreram no sexo masculino nas faixas etárias de 20-29 (192,0 a 262,0/100.000) anos e de 65 anos ou mais (188, 7 a 258,1/100.000). Os homicídios destacaram-se como primeira causa de morte em cerca de $75 \%$ dos distritos sanitários. Os autores discutem a necessidade de políticas públicas integradas e um conhecimento interdisciplinar para explicar as causas e enfrentar o problema da violência.

Palavras-chave Morte Súbita; Mortalidade; Violência; Análise Espacial 


\section{Introdução}

Nos últimos anos, a violência vem se constituindo em importante problema de saúde pública, não só pelos danos físicos e psíquicos que ela impõe, como também pelo número de óbitos ocasionados. Esse fato revela-se na magnitude da mortalidade por causas externas que ocupava, em 1995, o segundo lugar entre os grupos de causas de óbito no Brasil, superado apenas pelas doenças cardiovasculares (MS, 1996). Em estudo realizado em 15 países da América, entre 1971 e 1991, o Brasil situou-se entre aqueles que exibiam uma tendência de crescimento das taxas de mortalidade por causas externas, constituindo-se os acidentes de trânsito e homicídios nas principais causas de morte violenta (Yunes \& Rajs, 1994).

O perfil da mortalidade por causas externas no Brasil caracteriza-se por uma ocorrência maior nas regiões metropolitanas e faixas etárias mais jovens (Minayo, 1994). Na Bahia, a taxa de mortalidade por causas externas foi de 41,02/100.000 habitantes em 1995 (MS, 1996). Em Salvador, capital deste estado, verificou-se também um crescimento da mortalidade proporcional por esse grupo de causas a partir da segunda metade da década de 80 , bem como uma elevação de $22 \%$ nas taxas de mortalidade entre 1980-1989, inferior apenas àquelas observadas para Recife, São Paulo, Porto Alegre e Rio de Janeiro (Souza \& Minayo, 1995).

Os estudos realizados em Salvador na presente década apontam para a distribuição desigual do risco de morte violenta no espaço urbano, penalizando as áreas mais pobres da cidade (Paim \& Costa, 1996; CEDEC, 1997). Constata-se, ainda, um aumento das taxas de mortalidade por esse grupo de causas, quando se comparam os agregados de bairros com condições de vida intermediárias e aqueles de baixas e muito baixas condições de vida, especialmente no caso dos homicídios. (Almeida \& Paim, 1998).

Os trabalhos supracitados revelam, além dos diferenciais intra-urbanos de mortalidade por causas externas em Salvador, certas relações com as condições de vida dos residentes nas áreas mais afetadas, tal como fora observado, também, em Recife (Lima \& Ximenes, 1998). Caberia examinar, por conseguinte, a evolução dessa taxa nas diversas zonas da cidade, no sentido de identificar aquelas que experimentaram um crescimento e as que se beneficiaram com uma redução da mortalidade por causas externas nos últimos anos. O monitoramento da violência em um município, com base em dados secundários (Prefeitura Municipal de São
Paulo, 1992; SMS - Campinas, 1992), pode permitir avaliações futuras das medidas de controle porventura adotadas.

Desse modo, o objetivo deste trabalho é descrever a evolução da mortalidade por causas externas nas diversas zonas que compõem o espaço urbano de Salvador nos anos de 1988, 1991 e 1994.

\section{Material e métodos}

O presente estudo foi realizado valendo-se de óbitos dos residentes em Salvador, ocorridos em 1988, 1991 e 1994, e utilizou bases individual e ecológica como unidades de análise.

As informações sobre a mortalidade foram extraídas de bancos de dados do Instituto de Saúde Coletiva (ISC). Os dados originais para a composição desses bancos foram obtidos das Declarações de Óbito (DO) dos respectivos anos, fornecidas pelo Centro de Informações de Saúde da Secretaria de Saúde do Estado da Bahia (CIS/SESAB). Para os anos de 1991 e 1994, foram organizados bancos de dados complementares articulados aos anteriores, tendo como fontes o guia policial e o laudo técnico disponíveis nos arquivos do Instituto Médico Legal Nina Rodrigues (IMLNR).

Para possibilitar o estudo da distribuição espacial desses óbitos, o endereço de residência do falecido foi classificado segundo $75 \mathrm{zo}$ nas de informação (ZI), que correspondem a áreas da cidade estabelecidas pela CONDER (Companhia de Desenvolvimento da Região Metropolitana de Salvador), com base em critérios físico-urbanísticos, administrativos e de planejamento, compatibilizados com os setores censitários do IBGE (Fundação Instituto Brasileiro de Geografia e Estatística). Os procedimentos adotados para a codificação dos endereços nas distintas ZI encontram-se descritos em publicações anteriores (Paim et al., 1987; Paim \& Costa, 1993, 1996). Vale referir que as causas básicas de óbito já se encontravam selecionadas e codificadas pela SESAB, de acordo com a Classificação Internacional das Doenças (CID), nona revisão, correspondendo, portanto, à classificação suplementar das causas externas, de E800 a E999 (OPS, 1985).

Foram calculadas, para esse grupo de causas, a mortalidade proporcional (MP) e as taxas de mortalidade por sexo, faixa etária, e local de residência (ZI e distrito sanitário). As populações empregadas como denominadores das taxas foram obtidas do IBGE, para o ano de 1991, e de estimativas realizadas, para 1988 e 1994. 
Além do emprego das zonas de informação e dos distritos sanitários como unidades de análise da distribuição espacial, foram também utilizados agregados correspondentes a quartis de baixa, intermediária, elevada e muito elevada taxas de mortalidade por causas externas em 1988, cujos valores constituíram-se como parâmetros para o estudo da evolução em 1991 e 1994. Na análise por distrito sanitário, foram investigadas algumas causas específicas das mortes que compõem o grupo das causas externas apenas em 1991 e 1994, por serem os anos para os quais foi possível obter informações complementares no Instituto Médico Legal Nina Rodrigues sobre os tipos de mortes violentas. Os dados foram processados nos programas Epi-Info, versão 6.0 (Dean et al., 1994), e Epi-Map (Dean et al., 1995).

\section{Resultados}

No período de 1988 a 1994, em Salvador, o número absoluto de mortes provocadas pela violência variou entre 1.244, em 1988, e 1.674, em 1994, o que corresponde a um aumento de $34,6 \%$, encontrando-se em 1991 a maior taxa de mortalidade por esse grupo de causas: 77,9 por 100.000 habitantes (Figura 1).

Para os três anos, verificaram-se maiores taxas nos idosos com 65 anos e mais e na faixa etária de 20 a 29 anos, principalmente para o sexo masculino, casos em que os valores variaram de 188,7 a 262/100.000 habitantes. Entre os jovens de 15 a 19 anos, constata-se a terceira maior taxa em 1994 (em 1991 esta posição era da faixa de 40 a 49 anos, e a de 15 a 19 anos ocu- pava o quarto lugar). Em todos os grupos etários, o sexo masculino exibe maior risco de morte violenta, especialmente nos adultos jovens (Tabela 1). A sobremortalidade masculina variou entre quatro e seis vezes em relação à feminina. Foram os jovens de 15 a 19 anos e os idosos que apresentaram taxas crescentes no período, particularmente entre 1988 e 1991.

As maiores taxas de mortalidade por causas externas, nos anos de 1991 e 1994, foram verificadas nos distritos sanitários de São Caetano, Subúrbio Ferroviário, Cabula-Beiru, Liberdade

Figura 1

Mortalidade proporcional e taxa de mortalidade (por 100.000 habitantes) por causas externas. Salvador, 1988, 1991 e 1994

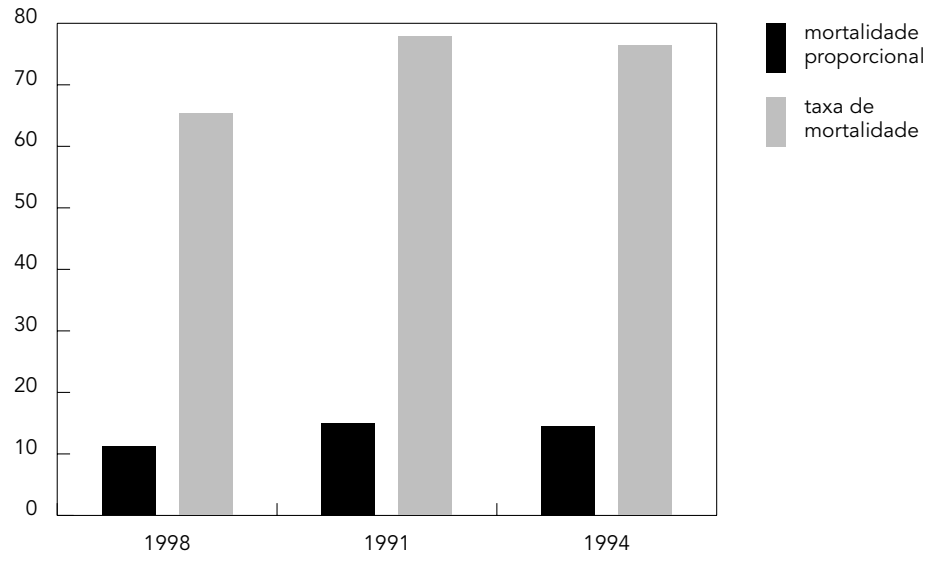

Tabela 1

Taxa de mortalidade (por 100.000 habitantes) por causas externas, segundo sexo e faixa etária.

Salvador, 1988, 1991 e 1994.

\begin{tabular}{lrrrrrrrrr}
\hline Faixa etária & & $\begin{array}{c}1988 \\
\text { Masc. }\end{array}$ & Total & Fem. & $\begin{array}{r}1991 \\
\text { Masc. }\end{array}$ & Total & $\begin{array}{r}\text { Fem. } \\
\text { Masc. } \\
\end{array}$ & Fem. & Total \\
\hline $0-9$ & 28,2 & 37,4 & 33,1 & 13,2 & 17,8 & 20,2 & 12,7 & 19,8 & 16,3 \\
$10-14$ & 13,6 & 29,9 & 21,6 & 8,5 & 36,3 & 22,2 & 17,5 & 27,9 & 22,6 \\
$15-19$ & 17,1 & 132,9 & 70,4 & 16,5 & 185,0 & 94,1 & 20,9 & 181,2 & 94,7 \\
$20-29$ & 22,2 & 192,0 & 100,7 & 23,4 & 262,0 & 133,7 & 14,5 & 228,6 & 113,5 \\
$30-39$ & 13,5 & 137,7 & 71,4 & 17,7 & 169,1 & 88,3 & 19,4 & 160,1 & 85,0 \\
$40-49$ & 18,5 & 132,9 & 72,8 & 33,0 & 163,9 & 94,4 & 25,3 & 138,5 & 78,4 \\
$50-64$ & 31,3 & 122,4 & 60,6 & 27,6 & 155,0 & 83,1 & 22,6 & 139,2 & 72,8 \\
$65+$ & 90,4 & 188,7 & 126,4 & 97,9 & 232,4 & 147,1 & 85,6 & 251,8 & 146,4 \\
Total & 23,9 & 111,6 & 65,3 & 22,4 & 140,2 & 77,9 & 21,1 & 132,2 & 75,4 \\
\hline
\end{tabular}


e Pau da Lima, variando entre 75,4 e 85/100.000 habitantes. Os homicídios constituíram-se na primeira causa de morte violenta em nove dos 12 distritos sanitários de Salvador em 1991, e em oito no ano de 1994. Observa-se, ainda, que, entre 1991 e 1994, houve um crescimento das taxas de mortalidade por esta causa específica para os residentes dos distritos do Subúrbio Ferroviário, Liberdade, São Caetano/Valéria, Pau da Lima, Cajazeiras, Itapagipe, Barra/Rio Vermelho e Boca do Rio, ocorrendo redução apenas nos distritos no Centro Histórico, Brotas, Itapuã e Pau da Lima. As mortes por acidentes de trânsito somente conseguem superar aquelas causadas pelos homicídios nos distritos de Barra/Rio Vermelho, Boca do Rio, Cajazeiras e Centro Histórico, neste último, apenas em 1994, e no primeiro deles só em 1991 (Tabela 2).

No caso dos suicídios, apesar da possível instabilidade dos pequenos números, alguns distritos sobressaem-se, como foi o caso do Centro Histórico, Liberdade e Cabula-Beiru, este comparecendo com a mais elevada taxa entre todos os distritos em 1994 (7,5/100.000). Com relação à queda como causa de óbito, os distritos do Centro Histórico e da Liberdade apareceram nos primeiros lugares, enquanto, no que se refere aos afogamentos, destacaramse os distritos de Itapuã, Cajazeiras e Boca do Rio, em 1991, São Caetano, em 1994, e Pau da Lima, nos dois anos em questão (Tabela 2).

No ano de 1988, em apenas 9,3\% das 75 zonas de informação (ZI), a mortalidade propor- cional por causas externas era igual ou superior a 20\%. Nos anos de 1991 e 1994, observa-se que esta proporção já é ultrapassada em 17,3\% e $21,3 \%$ das ZI, respectivamente, destacandose como mortalidade proporcional elevada nos três anos as ZI de Pernambués, Frederico Pontes, Fazenda Grande, Engomadeira, Mata Escura, Lobato/Pirajá, Sete de Abril, Escada/Periperi, Valéria, entre outros.

Elevados riscos dessas mortes nos três anos de estudo, com taxas de mortalidade na sua maioria superiores a 80/100.000 habitantes, ocorreram nas ZI do Rio Vermelho, Centro Histórico/Nazaré, Brotas, Frederico Pontes, Pernambués, Cabula, Liberdade, Fazenda Grande, São Gonçalo do Retiro, Lobato/Pirajá, Pau da Lima, Valéria (Tabela 3).

Na Figura 2, constata-se uma distribuição desigual de mortes violentas no espaço urbano de Salvador nos três anos em estudo, de modo que os residentes nas ZI correspondentes às áreas periféricas, como o Subúrbio Ferroviário e o chamado miolo da cidade (espaço situado entre a Rodovia BR-324 e a Avenida Paralela), apresentaram taxas incluídas nos quartis de elevada ou muito elevada mortalidade.

A evolução das mortes violentas em Salvador pode ser também examinada com base nas variações do comportamento de cada ZI no que se refere ao crescimento, manutenção ou diminuição das taxas (Tabelas 3 e 4). Assim, verifica-se que houve elevação destas (acima de $50 \%$ ) nas ZI de Jardim Apipema, Nordeste de

Tabela 2

Taxa de mortalidade (por 100.000 habitantes) por causas externas específicas, segundo distrito sanitário.

Salvador, 1991 e 1994

\begin{tabular}{|c|c|c|c|c|c|c|c|c|c|c|c|c|c|c|c|c|}
\hline \multirow[t]{2}{*}{$\begin{array}{l}\text { Distritos } \\
\text { Sanitários }\end{array}$} & \multicolumn{2}{|c|}{$\begin{array}{l}\text { Acidentes } \\
\text { de trânsito }\end{array}$} & \multicolumn{2}{|c|}{ Quedas } & \multicolumn{2}{|c|}{ Afogamentos } & \multicolumn{2}{|c|}{$\begin{array}{l}\text { Outros } \\
\text { acidentes }\end{array}$} & \multicolumn{2}{|c|}{ Suicídios } & \multicolumn{2}{|c|}{ Homicídios } & \multicolumn{2}{|c|}{ Intervenção legal } & \multicolumn{2}{|c|}{ Total } \\
\hline & 1991 & 1994 & 1991 & 1994 & 1991 & 1994 & 1991 & 1994 & 1991 & 1994 & 1991 & 1994 & 1991 & 1994 & 1991 & 1994 \\
\hline 1- C. Histórico & 22,7 & 18,9 & 12,6 & 5,9 & 2,5 & 1,2 & 2,5 & 2,4 & 5,0 & 5,9 & 29,0 & 15,3 & 3,8 & 3,5 & 89,5 & 60,1 \\
\hline 2- Itapagipe & 13,4 & 15,1 & 4,0 & 5,0 & 4,7 & 3,1 & 1,3 & 0,0 & 2,0 & 3,8 & 25,5 & 26,4 & 5,4 & 1,9 & 63,1 & 62,2 \\
\hline 3- S. Caetano & 23,1 & 21,1 & 2,6 & 3,4 & 4,6 & 7,2 & 2,1 & 0,5 & 2,1 & 3,8 & 34,4 & 38,9 & 6,7 & 5,8 & 84,7 & 85,0 \\
\hline 4- Liberdade & 18,8 & 11,4 & 8,8 & 7,7 & 6,1 & 3,6 & 1,7 & 2,6 & 3,9 & 2,1 & 21,0 & 37,7 & 7,2 & 4,1 & 77,8 & 75,4 \\
\hline 5- Brotas & 21,0 & 17,0 & 4,5 & 4,3 & 4,0 & 1,6 & 5,1 & 0,0 & 2,7 & 2,8 & 22,2 & 21,3 & 5,1 & 3,7 & 72,2 & 53,2 \\
\hline 6- Barra/R. Verm. & 20,0 & 15,2 & 5,7 & 3,0 & 4,5 & 4,2 & 2,2 & 0,6 & 5,1 & 3,0 & 19,7 & 21,3 & 2,5 & 3,0 & 68,3 & 45,5 \\
\hline 7- Boca do Rio & 22,2 & 25,3 & 2,5 & 3,5 & 9,9 & 2,3 & 4,9 & 1,2 & 2,5 & 3,5 & 12,3 & 15,0 & 2,5 & 1,2 & 59,1 & 53,0 \\
\hline 8- Itapuã & 23,5 & 20,5 & 3,0 & 4,2 & 10,6 & 2,8 & 1,5 & 1,4 & 1,5 & 2,8 & 32,5 & 20,5 & 4,5 & 2,8 & 84,4 & 58,8 \\
\hline 9- Cabula & 20,0 & 18,7 & 6,0 & 2,5 & 3,5 & 5,7 & 2,5 & 3,2 & 1,1 & 7,5 & 35,0 & 34,8 & 4,2 & 6,5 & 82,7 & 80,8 \\
\hline 10- Pau da Lima & 18,8 & 25,4 & 4,2 & 2,0 & 7,0 & 7,2 & 2,8 & 2,6 & 1,4 & 1,3 & 32,7 & 31,9 & 2,1 & 3,3 & 74,5 & 76,8 \\
\hline 11- Sub. Ferrov. & 24,7 & 24,5 & 2,3 & 2,8 & 4,6 & 3,9 & 1,9 & 2,5 & 2,7 & 1,1 & 25,5 & 38,0 & 11,4 & 5,0 & 83,6 & 82,8 \\
\hline 12- Cajazeiras & 19,0 & 20,6 & 5,0 & 3,7 & 10,0 & 5,6 & 1,0 & 1,9 & 3,0 & - & 17,0 & 19,6 & 5,0 & 3,7 & 69,0 & 57,1 \\
\hline Total & 21,1 & 20,2 & 5,0 & 3,8 & 5,5 & 4,5 & 2,4 & 1,8 & 2,8 & 3,4 & 26,6 & 29,4 & 5,5 & 4,5 & 77,9 & 75,4 \\
\hline
\end{tabular}


Causas externas - mortalidade proporcional, taxa de mortalidade (por 100.000 habitantes) e variação percentual da taxa de mortalidade segundo zona de informação. Salvador, 1988, 1991 e 1994.

\begin{tabular}{|c|c|c|c|c|c|c|c|c|c|}
\hline \multirow[t]{2}{*}{ Zona de informação } & \multicolumn{3}{|c|}{ Mortalidade proporcional } & \multicolumn{3}{|c|}{ Taxa de mortalidade } & \multicolumn{3}{|c|}{ Variação percentual } \\
\hline & 88 & 91 & 94 & 88 & 91 & 94 & $88-91$ & $88-94$ & $91-94$ \\
\hline 1- Barra & 5,1 & 8,3 & 6,1 & 31,2 & 45,1 & 30,6 & 44,3 & $-1,8$ & $-32,0$ \\
\hline 2- Jardim Apipema & 10,3 & 8,6 & 12,0 & 30,8 & 35,3 & 59,5 & 14,8 & 93,3 & 68,3 \\
\hline 3- Ondina & 9,7 & 11,1 & 10,0 & 70,2 & 86,0 & 80,4 & 22,5 & 14,5 & $-6,5$ \\
\hline 4/5- Rio Vermelho & 12,6 & 12,9 & 15,6 & 90,3 & 82,9 & 94,8 & $-8,1$ & 5,0 & 14,3 \\
\hline 6- Amaralina & 14,5 & 8,3 & 9,5 & 109,1 & 6,6 & 18,1 & $-94,0$ & $-83,4$ & 175,7 \\
\hline 7- Nordeste Amaralina & 10,4 & 8,6 & 11,6 & 39,6 & 134,0 & 70,1 & 238,7 & 77,2 & $-47,7$ \\
\hline 8- Pituba & 8,5 & 12,7 & 9,0 & 48,1 & 82,5 & 46,8 & 71,5 & $-2,6$ & $-43,2$ \\
\hline 9- Campo Grande/Vitória & 10,3 & 11,9 & 6,3 & 41,2 & 88,3 & 47,2 & 114,3 & 14,5 & $-46,6$ \\
\hline 10- Graça & 8,3 & 11,8 & 5,5 & 74,8 & 76,3 & 42,8 & 2,1 & $-42,7$ & $-43,9$ \\
\hline 11/12- Alto das Pombas & 12,0 & 12,6 & 13,1 & 73,9 & 66,5 & 63,5 & $-10,1$ & $-14,1$ & $-4,4$ \\
\hline 13- Horto Florestal & 8,7 & 14,3 & 20,0 & 16,0 & 7,4 & 13,8 & $-54,1$ & $-14,1$ & 87,0 \\
\hline 14- Canela & 9,8 & 8,1 & 41,9 & 72,0 & 49,6 & 0,0 & $-31,1$ & $-100,0$ & $-100,0$ \\
\hline 15/25- Centro Histórico/Nazaré & 7,2 & 11,6 & 8,2 & 86,7 & 128,7 & 94,6 & 48,4 & 9,0 & $-26,5$ \\
\hline 16- Garcia & 8,3 & 9,1 & 6,2 & 76,9 & 70,6 & 33,0 & $-8,1$ & $-57,0$ & $-53,2$ \\
\hline 17- Barra/F. Nova & 7,2 & 5,0 & 10,8 & 56,3 & 22,1 & 48,3 & $-60,6$ & $-14,1$ & 118,2 \\
\hline 18- Eng. V. de Brotas & 12,3 & 18,7 & 10,0 & 63,3 & 88,8 & 40,1 & 40,2 & $-36,7$ & $-54,8$ \\
\hline 19- Acupe & 6,8 & 15,4 & 6,8 & 12,4 & 30,3 & 10,6 & 144,9 & $-14,1$ & $-64,9$ \\
\hline 20-Brotas & 8,2 & 9,3 & 8,3 & 99,7 & 115,2 & 82,9 & 15,2 & $-16,9$ & $-28,0$ \\
\hline 21- Pq. N. S. Luz/Itaigara & 15,1 & 22,4 & 7,4 & 28,9 & 36,5 & 6,2 & 26,3 & $-78,5$ & $-83,0$ \\
\hline 22- Stiep/Armação & 5,2 & 13,2 & 11,0 & 18,8 & 38,8 & 32,3 & 106,7 & 71,8 & $-16,9$ \\
\hline 23- Frederico Pontes & 26,3 & 18,7 & 25,0 & 501,5 & 276,5 & 86,2 & $-44,9$ & $-82,8$ & $-68,8$ \\
\hline 24- R. Chile/Baixa dos Sapateiros & 11,8 & 9,9 & 10,5 & 105,3 & 62,2 & 25,8 & $-41,0$ & $-75,5$ & $-58,4$ \\
\hline 26- Matatu & 8,7 & 8,5 & 7,1 & 82,0 & 75,3 & 56,3 & $-8,1$ & $-31,3$ & $-25,2$ \\
\hline 27- Cosme de Farias & 8,8 & 9,5 & 20,2 & 61,2 & 98,4 & 118,3 & 60,7 & 93,3 & 20,2 \\
\hline 28- Luis Anselmo/Vila Laura & 12,8 & 17,8 & 8,9 & 26,5 & 24,4 & 18,2 & $-7,9$ & $-31,3$ & $-25,4$ \\
\hline 29- Cabula & 10,5 & 13,8 & 17,1 & 70,0 & 92,4 & 146,6 & 32,0 & 109,4 & 58,6 \\
\hline 30- Pernambués & 16,4 & 18,2 & 20,8 & 115,3 & 105,9 & 122,3 & $-8,1$ & 7,8 & 17,4 \\
\hline 31- Boca do Rio & 9,9 & 15,8 & 15,2 & 46,2 & 80,2 & 83,8 & 73,5 & 81,4 & 4,5 \\
\hline 32- Barbalho/Lapinha & 5,0 & 9,1 & 6,7 & 49,6 & 79,7 & 53,3 & 60,7 & 7,4 & $-33,2$ \\
\hline 33- Caixa d'Água & 11,4 & 7,2 & 13,1 & 48,8 & 24,1 & 41,9 & $-50,5$ & $-14,1$ & 73,7 \\
\hline 34- Quintas/Cidade Nova & 13,3 & 11,8 & 9,8 & 53,1 & 71,9 & 60,0 & 35,4 & 13,0 & $-16,5$ \\
\hline 35- Acesso Norte & - & 25,0 & - & - & $1.342,3$ & - & - & - & $-100,0$ \\
\hline 36- $19 \mathrm{BC}$ & - & 18,9 & - & 31,8 & 34,1 & - & 7,2 & $-100,0$ & $-100,0$ \\
\hline 37- Pituaçu & 15,6 & 15,6 & 20,0 & 48,7 & 32,0 & 35,9 & $-34,4$ & $-26,4$ & 12,2 \\
\hline 38-Calçada/Mares & 9,0 & 11,2 & 7,5 & 84,0 & 100,9 & 66,6 & 20,1 & $-20,7$ & $-34,0$ \\
\hline 39/40- Liberdade & 12,8 & 13,1 & 15,3 & 99,6 & 98,9 & 95,9 & 0,7 & $-3,7$ & $-3,0$ \\
\hline 41- IAPI & 11,1 & 11,8 & 17,0 & 71,9 & 62,9 & 94,1 & $-12,5$ & 30,9 & 49,6 \\
\hline 42- Fazenda Grande & 16,1 & 14,4 & 19,5 & 125,8 & 93,4 & 170,1 & $-25,7$ & 35,3 & 82,1 \\
\hline 43- S. G. do Retiro & 15,2 & 27,0 & 6,4 & 97,6 & 128,9 & 15,7 & 32,0 & $-83,9$ & $-87,8$ \\
\hline 44- Engomadeira & 25,2 & 23,4 & 25,2 & 59,0 & 108,3 & 102,5 & 83,7 & 73,9 & $-5,3$ \\
\hline 45- $C A B$ & 50,0 & - & - & 39,3 & - & - & $-100,0$ & $-100,0$ & - \\
\hline 46- Piatã/Patamares & 33,4 & 13,0 & 16,0 & 46,5 & 64,0 & 79,8 & 37,8 & 71,8 & 24,7 \\
\hline 47- Bonfim/Ribeira & 7,6 & 5,3 & 8,7 & 88,2 & 45,8 & 85,6 & $-48,1$ & $-2,9$ & 87,0 \\
\hline 48- Jardim Cruzeiro & 9,7 & 16,1 & 10,6 & 51,4 & 75,5 & 44,1 & 47,0 & $-14,1$ & $-41,5$ \\
\hline 49- Uruguai & 11,3 & 11,0 & 16,9 & 64,4 & 47,3 & 77,5 & $-26,5$ & 20,3 & 63,7 \\
\hline 50/51- São Caetano & 8,9 & 17,6 & 15,5 & 49,5 & 83,9 & 58,8 & 69,6 & 18,9 & $-29,9$ \\
\hline 52- Mata Escura & 18,0 & 17,5 & 21,2 & 78,0 & 87,2 & 72,9 & 11,8 & $-6,6$ & $-16,5$ \\
\hline 53- Sussuarana & 12,3 & 22,4 & 22,0 & 38,5 & 61,3 & 59,6 & 59,2 & 54,6 & $-2,9$ \\
\hline
\end{tabular}

(continua) 


\begin{tabular}{|c|c|c|c|c|c|c|c|c|c|}
\hline \multirow[t]{2}{*}{ Zona de informação } & \multicolumn{3}{|c|}{ Mortalidade proporcional } & \multicolumn{3}{|c|}{ Taxa de mortalidade } & \multicolumn{3}{|c|}{ Variação do percentual } \\
\hline & 88 & 91 & 94 & 88 & 91 & 94 & $88-91$ & $88-94$ & $91-94$ \\
\hline 54- Paralela/E. Velha do Aeroporto & 11,5 & 16,3 & 13,0 & 30,4 & 30,0 & 34,1 & $-1,1$ & 12,3 & 13,6 \\
\hline 55- Itapuã & 14,0 & 24,8 & 15,2 & 68,8 & 118,3 & 69,7 & 71,9 & 1,3 & $-41,1$ \\
\hline 56- P. do Flamengo & 20,0 & - & 16,7 & 52,8 & - & 45,4 & $-100,0$ & $-14,1$ & - \\
\hline 57- Lobato/Pirajá & 15,7 & 22,3 & 23,8 & 96,7 & 107,0 & 121,4 & 10,7 & 25,6 & 13,4 \\
\hline 58- Campinas & 17,3 & 20,5 & 15,4 & 82,4 & 31,9 & 29,8 & $-61,3$ & $-63,8$ & $-6,5$ \\
\hline 59- Pau da Lima & 14,3 & 17,6 & 15,6 & 96,4 & 107,2 & 82,8 & 11,2 & $-14,1$ & $-22,7$ \\
\hline 60- Sete de Abril & 11,0 & 16,1 & 20,3 & 65,5 & 150,3 & 178,1 & 129,6 & 172,0 & 18,5 \\
\hline 61- Mussurunga/S. Cristóvão & 11,0 & 11,8 & 16,9 & 39,3 & 74,5 & 73,9 & 89,4 & 88,1 & $-0,7$ \\
\hline 62- Plataforma & 12,9 & 16,6 & 18,0 & 89,7 & 91,0 & 80,3 & 1,4 & $-10,5$ & $-11,8$ \\
\hline 63- Pirajá & 12,7 & 20,2 & 17,8 & 81,1 & 96,4 & 94,2 & 18,9 & 16,2 & $-2,3$ \\
\hline 64- Pirajá/P. Seco & 27,3 & 18,7 & 25,6 & 70,5 & 32,4 & 101,0 & $-54,1$ & 43,2 & 211,8 \\
\hline 65- Castelo Branco & 7,9 & 11,4 & 16,5 & 34,9 & 56,2 & 60,0 & 61,0 & 71,9 & 6,8 \\
\hline 66- Escada/Periperi & 10,1 & 16,1 & 16,4 & 78,5 & 116,1 & 129,2 & 48,0 & 64,7 & 11,3 \\
\hline 67- São Bartolomeu & - & 36,4 & 60,0 & - & 49,4 & 69,3 & - & - & 40,3 \\
\hline 68-Valéria & 15,1 & 23,6 & 21,2 & 150,7 & 207,6 & 194,2 & 37,8 & 28,9 & $-6,5$ \\
\hline 69- Águas Claras & 16,5 & 21,9 & 21,2 & 45,7 & 69,0 & 58,0 & 50,9 & 26,8 & $-16,0$ \\
\hline 70- Est. Cia/Aerop. & 21,4 & 14,3 & 25,0 & 16,9 & 15,5 & 19,3 & $-8,1$ & 14,5 & 24,7 \\
\hline 71- Coutos & 8,0 & 18,0 & 16,6 & 36,6 & 49,6 & 47,9 & 35,6 & 30,9 & $-3,5$ \\
\hline 72- Limite c/Usiba & - & - & - & - & - & - & - & - & - \\
\hline 73- Paripe/Base Naval & 8,4 & 10,7 & 15,0 & 67,5 & 68,9 & 83,8 & 2,1 & 24,1 & 21,6 \\
\hline 74- Iha de Bom Jesus/Frades & 11,8 & 25,0 & 14,3 & 61,8 & 56,7 & 53,1 & $-8,2$ & $-14,1$ & $-6,5$ \\
\hline 76- Ilha de Maré & - & - & - & - & - & - & - & - & - \\
\hline Total & 11,2 & 15,0 & 14,4 & 65,3 & 77,9 & 75,4 & 19,3 & 15,5 & $-3,2$ \\
\hline
\end{tabular}

Amaralina, Stiep/Armação, Cosme de Farias, Cabula, Boca do Rio, Piatã/Patamares, Sussuarana, Sete de Abril, Mussurunga/São Cristovão, Castelo Branco e Escada/Periperi. Contudo, as ZI que apresentaram maior crescimento das taxas entre os períodos de 1988-1991, 19911994 e 1988-1994 foram Nordeste de Amaralina (238,7\%), Pirajá/Porto Seco $(211,8 \%)$ e Sete de Abril (172,0\%), respectivamente. No último período, houve um decréscimo mais acentuado (50\%-99\%) nas ZI de Amaralina, Canela, Garcia, Parque Nossa Senhora da Luz/Itaigara, Frederico Pontes, Rua Chile/Baixa dos Sapateiros, 19 BC, São Gonçalo do Retiro, Centro Administrativo da Bahia e Campinas.

\section{Discussão}

A tendência de crescimento no número de óbitos e no risco absoluto das mortes por causas externas em Salvador, de 1988 para 1994, torna evidente a importância da violência no perfil de mortalidade, razão pela qual essa cidade encontra-se entre as capitais com taxas mais altas que a média do País (Laurenti \& Mello-Jorge, 1997).
A taxa de mortalidade até seis vezes maior para o sexo masculino indica uma também maior exposição dos homens a mortes violentas, perfil igualmente observado para o Brasil no final da década de 80 (Minayo, 1994). Do mesmo modo, as maiores taxas verificadas nos idosos e adultos jovens coincidem com o padrão de distribuição segundo sexo e idade, observado não só no Brasil, como em outros países, tais como Itália, Israel e Alemanha (Mello-Jorge et al., 1997; Lima \& Ximenes, 1998). As elevadas taxas observadas em maiores de 65 anos de ambos os sexos podem estar relacionadas com uma maior vulnerabilidade desse grupo etário aos acidentes (atropelos, quedas etc.), do ponto de vista tanto biológico, quanto psicológico e social.

O crescimento do risco no grupo de 10-19 anos e do aumento da proporção de óbitos na faixa etária de 15-19 anos, que passou do quinto grupo mais acometido por mortes violentas em 1988, para o terceiro em 1994, reafirma a importância das mortes violentas nesse grupo etário (Mello-Jorge \& Marques, 1988; Minayo, 1990), principalmente em decorrência dos homicídios (Mello-Jorge et al., 1997).

As mortes violentas distribuem-se de forma heterogênea nas zonas de informação (ZI) 


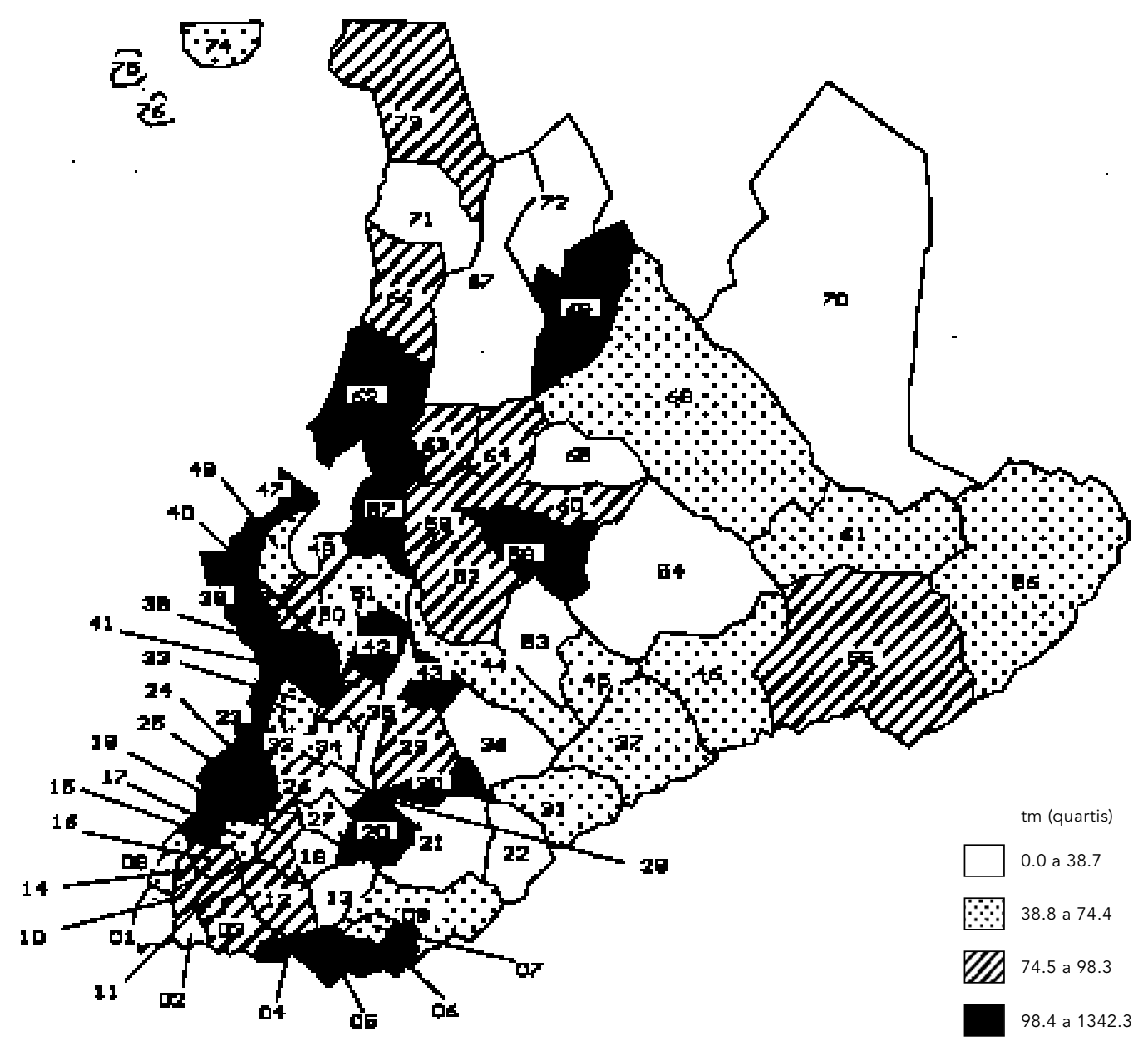

que compõem o Município de Salvador. As áreas que apresentaram indicadores mais elevados, com taxas superiores à média da cidade, corresponderam, na maioria das vezes, a bairros com precárias condições de vida (CEDEC, 1997). É o caso, por exemplo, das ZI de Valéria e Sete de Abril, que igualmente apresentaram taxas elevadas de mortalidade para a faixa etária de crianças e adolescentes em Salvador, em 1991 (Paim \& Costa, 1996). Em relação à mortalidade proporcional, as ZI que compõem o Subúrbio Ferroviário e as que se localizam mais na periferia da cidade apresentaram os maiores valores, enquanto o contrário foi observado nas áreas mais nobres, localizadas no centro e costa litorânea, indo da Barra a Pituaçu.

Os homicídios constituíram-se no tipo mais freqüente de morte violenta em cerca de $75 \%$ dos 12 distritos sanitários de Salvador, ultrapassando os acidentes de trânsito e apresentando um crescimento das taxas entre $1991 \mathrm{e}$ 1994. Tendência semelhante vem sendo verificada no resto do Brasil e no México (Minayo, 1994; Souza, 1994; Lopez \& Ramon, 1996). Essa informação precisa ser considerada pelas administrações regionais da Prefeitura de Salvador, pelos órgãos de segurança pública do Estado, e, particularmente, pelas gerências dos distritos sanitários. 
Distribuição espacial da taxa de mortalidade (por 100.000 habitantes) por causas externas, segundo zonas de informação. Salvador, 1991.

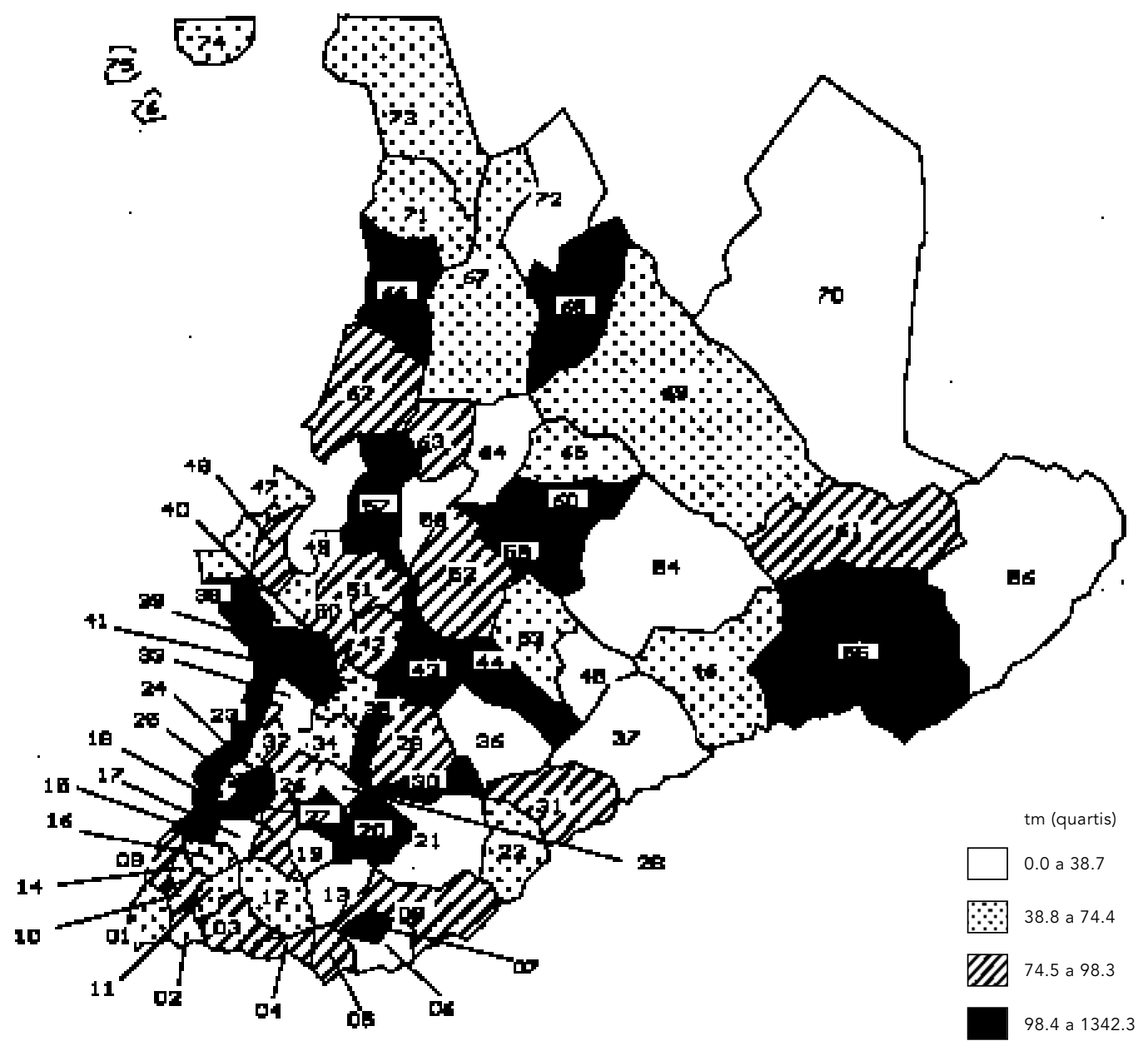

O reconhecimento da violência como problema de saúde pública (OPS, 1996) deve ser ressaltado entre os trabalhadores de saúde para superar situações como aquela observada durante a formulação do Plano Municipal de Saúde (1988-2001), quando apenas o Distrito Barra-Rio Vermelho admitiu a violência como problema prioritário de saúde, apesar de os indicadores disponíveis apresentaram-na como importante causa de morte também para os demais distritos (SMS-Salvador, 1997).

Os serviços de saúde, por meio da utilização da epidemiologia, podem contribuir na produção de evidências que apontem para fatores envolvidos na geração da violência e na identificação daqueles tipos que mais atingem a população em seus diferentes estratos sociais, território, faixa etária e sexo, visando à adoção de medidas de prevenção e controle melhor fundamentadas, inteligentes e criativas. Nesse particular, devem estar comprometidos não só o setor da saúde, mas outros segmentos organizados da sociedade e o cidadão comum, que podem contribuir na redução da morbi-mortalidade por causas externas e dos riscos da violência. Se os determinantes da violência e do seu crescimento envolvem aspectos econômicos, sociais, políticos, culturais, a resposta social organizada a essa epidemia requer um plano de ação bem-estruturado, além de 


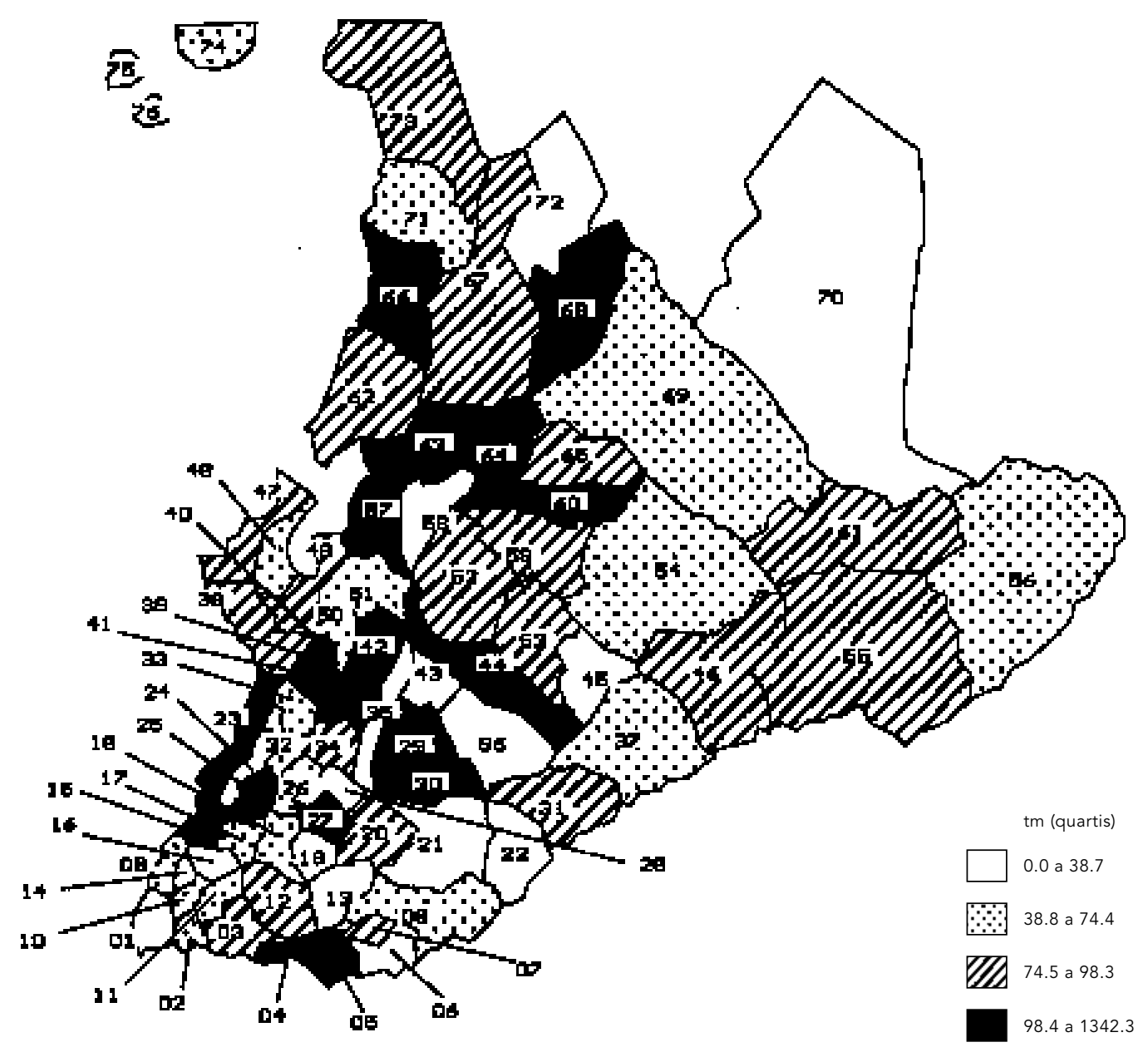

mudanças sócio-econômicas, tal como vem sendo recomendado por pesquisadores e por organismos nacionais e internacionais (Minayo \& Souza, 1993; OPS, 1993; Minayo, 1994; OPS, 1996; Paim \& Costa, 1996; CEDEC, 1997; Laurenti \& Mello-Jorge, 1997). Por esses motivos, um estudo ecológico como este pode auxiliar na discriminação de áreas de maior risco, orientando a ação programática de distritos sanitários na prevenção e atenção de acidentes e violências e, especialmente, a adoção de estratégias intersetoriais de controle da morbi-mortalidade por causas externas.

Muitas das causas de mortes violentas consideradas acidentais são previsíveis e como tal, podem ser evitadas. Assim, os acidentes de trânsito, que, ao lado dos homicídios, são um dos principais tipos de mortes violentas no Brasil, vêm decrescendo, desde 1966, nos Estados Unidos, em decorrência de medidas de segurança tomadas no trânsito das cidades e estradas (Mello-Jorge et al., 1997). O Brasil dispõe, desde 1998, de um Código Nacional de Trânsito que, apesar das controvérsias (Nunes, 1998), pode contribuir significativamente para a redução dos mortos e feridos e para a instauração de uma convivência mais civilizada entre motoristas e pedestres. Não basta, porém, essa contribuição do Legislativo. Os cidadãos precisam conscientizar-se da pertinência do cum- 
Evolução da taxa de mortalidade (por 100.000 habitantes) por causas externas, segundo zonas de informação de Salvador, 1988-1991, 1991-1994 e 1988-1994.

\begin{tabular}{|c|c|c|c|}
\hline Zona de informação & $88-91$ & $91-94$ & $88-94$ \\
\hline 1- Barra & ++ & -- & - \\
\hline 2- Jardim Apipema & ++ & +++ & +++ \\
\hline 3- Ondina & ++ & - & ++ \\
\hline 4/5- Rio Vermelho & - & ++ & + \\
\hline 6- Amaralina & $-\cdots$ & ++++ & -- \\
\hline 7- Nordeste Amaralina & ++++ & -- & +++ \\
\hline 8- Pituba & +++ & -- & - \\
\hline 9- Campo Grande/Vitória & ++++ & -- & ++ \\
\hline 10- Graça & + & -- & -- \\
\hline 11/12- Federação/Alto das Pombas & -- & - & -- \\
\hline 13- Horto Florestal & $-\cdots$ & +++ & -- \\
\hline 14- Canela & -- & -- & $--\cdot$ \\
\hline 15/25- Centro Histórico/Nazaré & ++ & -- & + \\
\hline 16- Garcia & - & --- & --- \\
\hline 17- Barra/F. Nova & $-\cdots$ & ++++ & -- \\
\hline 18- Eng. V. Brotas & ++ & $-\cdots$ & -- \\
\hline 19- Acupe & ++++ & - - - & - \\
\hline 20- Brotas & ++ & -- & -- \\
\hline 21- Pq. N. S. Luz/Itaigara & ++ & -- & --- \\
\hline 22- Stiep/Armação & ++++ & -- & +++ \\
\hline 23- Frederico Pontes & -- & - - - & --- \\
\hline 24- Chile/Baixa dos Sapateiros & -- & - - - & $-\ldots$ \\
\hline 26- Matatu & - & -- & -- \\
\hline 27- Cosme de Farias & +++ & ++ & +++ \\
\hline 28- Luis Anselmo/Vila Laura & - & - & -- \\
\hline 29- Cabula & ++ & +++ & +++ \\
\hline 30- Pernambués & - & ++ & + \\
\hline 31- Boca do Rio & +++ & + & +++ \\
\hline 32- Barbalho/Lapinha & +++ & -- & + \\
\hline 33- Caixa d'Água & -- & +++ & -- \\
\hline 34- Quintas Cidade Nova & ++ & -- & ++ \\
\hline \multicolumn{4}{|l|}{ 35- Acesso Norte } \\
\hline 36- $19 \mathrm{BC}$ & + & - - - & -- \\
\hline 37- Pituaçu & - & ++ & -- \\
\hline 38- Calçada/Mares & ++ & - & - \\
\hline 39/40- Liberdade & -- & - & -- \\
\hline 41- IAPI & - - & ++ & ++ \\
\hline 42- Fazenda Grande & -- & +++ & ++ \\
\hline 43- S. G. do Retiro & ++ & $-\ldots$ & $-\cdots$ \\
\hline 44- Engomadeira & +++ & - & +++ \\
\hline 45- $C A B$ & -- & - - - & \\
\hline 46- Piatã/Patamares & ++ & ++ & +++ \\
\hline 47- Bonfim/Ribeira & -- & +++ & - \\
\hline 48- Jardim Cruzeiro & ++ & -- & -- \\
\hline 49- Uruguai & -- & +++ & ++ \\
\hline 50/51- São Caetano & +++ & -- & ++ \\
\hline 52- Mata Escura & ++ & - & - \\
\hline 53- Sussuarana & +++ & - & +++ \\
\hline
\end{tabular}

(continua) 
Tabela 4 (continuação)

\begin{tabular}{|c|c|c|c|}
\hline Zona de informação & $88-91$ & $91-94$ & $88-94$ \\
\hline 54- Paralela/E. Velha do Aeroporto & - & ++ & ++ \\
\hline 55- Itapuã & +++ & -- & + \\
\hline 56- P. Flamengo/S. Mares & - - - & & -- \\
\hline 57- Lobato/Pirajá & ++ & ++ & ++ \\
\hline 58- Campinas & - & - & - . - \\
\hline 59- Pau da Lima & ++ & -- & - \\
\hline 60- Sete de Abril & ++++ & ++ & ++++ \\
\hline 61- Mussurunga/S. Cristóvão & +++ & - & +++ \\
\hline 62- Plataforma & + & -- & -- \\
\hline 63- Pirajá & ++ & - & ++ \\
\hline 64- Pirajá/P. Seco & - & ++++ & ++ \\
\hline 65- Castelo Branco & +++ & + & +++ \\
\hline 66- Escada/Periperi & ++ & ++ & +++ \\
\hline 67- São Bartolomeu & & ++ & \\
\hline 68- Valéria & ++ & - & ++ \\
\hline 69- Águas Claras & +++ & - - & ++ \\
\hline 70- E. Cia/Aerop. & - & ++ & ++ \\
\hline 71- Coutos & ++ & - & ++ \\
\hline 72- Limite c/Usiba & $=$ & $=$ & $=$ \\
\hline 73- Paripe/Base Naval & + & ++ & ++ \\
\hline 74- Ilha Bom Jesus & - & - & - \\
\hline 76- Ilha de Maré & $=$ & $=$ & $=$ \\
\hline
\end{tabular}

(+) Crescimento $<10 \%$; (-) diminuição $<10 \%$.

(++) Crescimento entre 10,1-50,0\%; (- -) diminuição entre $10,1-50,0 \%$.

$(+++)$ Crescimento entre 50,1-100,0\%; (- - -) diminuição entre 50,1-100,0\%.

$(++++)$ Crescimento acima de 100,1\%; (=) sem alteração da taxa.

primento da legislação; o Executivo deve cumprir o seu papel fiscalizador e o Judiciário tem de garantir, com agilidade, a concretização da justiça.

Enquanto esforços se desenvolvem para o alcance desses propósitos, as Secretarias e Ministério da Saúde precisam exercer uma liderança institucional e setorial para o enfrentamento da morbi-mortalidade das causas externas, adotando, a exemplo da vigilância da saúde (OPS, 1993; Paim, 1993), modelos de atenção alternativos centrados na ação intersetorial sobre o território, valendo-se da utilização de conhecimentos e métodos tanto da epidemiologia, quanto das ciências sociais. Como foi destacado em outra oportunidade, a mortalidade por causas externas demanda um con- junto de medidas de controle que não pode ser monopólio da medicina, da justiça ou da segurança pública. Nesse sentido, "a Saúde Coletiva, o Planejamento Urbano, as Ciências Sociais, a Psicologia, a Pedagogia, o Direito, entre outros, são saberes que precisam se articular para informar práticas técnicas e sociais com o mais alto grau de efetividade sobre tais problemas" (Paim \& Costa, 1996:63-64). Estabelecer um diálogo com as crenças e ideologias sobre o tema sem negligenciar a busca de evidências, do conhecimento científico acumulado e de teorias fundamentadas pode ser um dos caminhos a serem explorados enquanto a publicidade, a arte e outras manifestações culturais reforçam os valores da paz, solidariedade e dignidade entre os seres humanos.

\section{Agradecimentos}

Trabalho realizado com o apoio da Organização Panamericana de Saúde/Conselho de Desenvolvimento Científico e Tecnológico, DRC/ RPD/63/5/12. Processo no 52.1820/93.6 (CNPq). 


\section{Referências}

ALMEIDA, L. M. A. \& PAIM, J. S., 1998. Análise da mortalidade por homicídios em Salvador segundo condições de vida em 1991 e 1994. In: 4o Congresso Brasileiro de Epidemiologia, Resumos, p. 37, Rio de Janeiro: ABRASCO.

MS (Ministério da Saúde), 1996. Estatísticas de Mortalidade. CD-ROM, Brasília: MS.

CEDEC (Centro de Estudos da Cultura Contemporânea), 1997. Mapa de Risco da Violência: Cidade de Salvador. São Paulo: CEDEC.

LAURENTI, R. \& MELLO-JORGE, M. H. P., 1997. Acidentes e violência no Brasil - Apresentação. Revista de Saúde Pública, 31 (Sup. 4):1-4.

LIMA, M. L. C. \& XIMENES, R., 1998. Violência e morte: Diferenciais da mortalidade por causas externas no espaço urbano do Recife. Cadernos de Saúde Pública, 14:829-840.

LOPEZ, M. V. \& RAMON, M. C. H. M., 1996. Muertes por homicidio, consecuencia fatal de la violencia. El caso de México, 1979-1992. Revista de Saúde Pública, 30:46-52.

MELLO-JORGE, M. H. P.; GAWRYSZEWSKI, V. P. \& LATORRE, M. R. D. D., 1997. Acidentes e violência no Brasil - Análise dos dados de mortalidade. Revista de Saúde Pública, 31 (Sup. 4):5-25.

MELLO-JORGE, M. H. P. \& MARQUES, M. B., 1988. Mortes violentas em menores de 15 anos no Brasil. Boletín de la Oficina Sanitaria Panamericana, 100:590-605.

MINAYO, M. C. S., 1990. A violência na adolescência: Um problema de saúde pública. Cadernos de Saúde Pública, 6:278-292.

MINAYO, M. C. S., 1994. A violência social sob a perspectiva da saúde pública. Cadernos de Saúde Pública, 10:7-18.

MINAYO, M. C. S. \& SOUZA, E. R., 1993. Violência para todos. Cadernos de Saúde Pública, 9:65-78.

NUNES, E., 1998. Autoridades de trânsito não respeitam novo código. Folha de São Paulo, São Paulo, 19 set., Caderno 3, p. 2.

OPS (Organización Panamericana de la Salud), 1993. Sobre la Teoria y Práctica de la Salud Pública. Un Debate, Múltiples Perspectivas. Washington, D.C.: OPS.

OPS (Organización Panamericana de la Salud), 1996. Sociedad, Violencia y Salud. Washington, D.C.: OPS.
OPS (Organización Panamericana de la Salud), 1985. Classificação Internacional das Doenças. Revisão 1975. Centro da OMS para Classificação de Doenças em Português. São Paulo: Ministério da Saúde/ Universidade de São Paulo/Organização Pan-Americana da Saúde.

PAIM, J. S., 1993. A reforma sanitária e os modelos assistenciais. In: Epidemiologia e Saúde (M. Z. Rouquayrol, org.), pp. 455-466, Rio de Janeiro: MEDSI.

PAIM, J. S. \& COSTA, M. C. N., 1993. Decréscimo e desigualdade da mortalidade infantil: Salvador, 1980-1988. Boletín de la Oficina Sanitaria Panamericana, 114:415-428.

PAIM, J. S. \& COSTA, M. C. N., 1996. Mortes violentas em crianças e adolescentes de Salvador. Bahia Análise e Dados, 6:59-67.

PAIM, J. S.; COSTA, M. C. N.; CABRAL, V.; MOTA, I. A. \& NEVES, R. B. B., 1987. Mortalidade infantil proporcional, Salvador, Bahia. Boletín de la Oficina Sanitaria Panamericana,103:113-122.

PREFEITURA MUNICIPAL DE SÃO PAULO, 1992. PRO-AIM/Programa de Aprimoramento das Informações de Mortalidade no Município de São Paulo. São Paulo: Prefeitura Municipal de São Paulo.

SMS-CAMPINAS (Secretaria Municipal de Saúde de Campinas), 1992. Mortalidade em Campinas. Informe Trimestral do Projeto de Monitorização dos Óbitos no Município de Campinas, Boletim no 6 .

SMS-SALVADOR (Secretaria Municipal de Saúde de Salvador), 1997. Plano Municipal de Saúde 19982001. Salvador: Prefeitura Municipal.

SOUZA, E. R., 1994. Homicídios no Brasil: O grande vilão da saúde pública na década de 80 . Cadernos de Saúde Pública, 10 (Sup. 1):45-60.

SOUZA, E. R. \& MINAYO, M. C. S., 1995. O impacto da violência social na saúde pública do Brasil: Década de 80. In: Os Muitos Brasis: Década de 80 (M. C. S. Minayo, org.), pp. 87-116, São Paulo: Editora Hucitec/Rio de Janeiro: ABRASCO

YUNES, J. \& RAJS, D., 1994. Tendencia de la mortalidad por causas violentas en la población general y entre los adolescentes y jóvenes de la región de las Américas. Cadernos de Saúde Pública, 10:4560. 